\title{
Cross-Education Effects of Muscle Strength and Balance on Unilateral Isokinetic Exercise in Ankle
}

\author{
Sung Min Son ${ }^{1}$, Kyung Woo Kang ${ }^{2}$ \\ ${ }^{1}$ Department of Physical Therapy, College of Health Science, Cheongju University, Cheongju; ${ }^{2}$ Department of Physical Therapy, Yeungnam University \\ College, Daegu, Korea
}

Purpose: Unilateral strength training effects on contralateral sides have been demonstrated in previous studies for lower extremity exercise, upper extremity exercise, and unilateral surface electrical stimulation. This study was performed to investigate the effects of unilateral ankle training on muscle strength and the balance of contralateral lower extremity in healthy adults.

Methods: Thirty healthy subjects were randomized equally to a training or a control group. Those in the training group received unilateral ankle isokinetic strengthening training of the dominant leg (right side) for 4 weeks. Contralateral single-limb balance, including Anterio-Posterior Stability Index (APSI), Medio-Lateral Stability Index (MLSI) and Overall Stability Index (OSI), was assessed before and after intervention.

Results: Comparison of pre- and post-test data revealed significant improvements in ipsi- and contralateral ankle strengths, and significant improvement in contralateral single limb balance.

Conclusion: These results have practical implications because they demonstrate that unilateral ankle isokinetic exercise improves ankle muscle strength and balance ability of contralateral lower extremity.

Keywords: Unilateral ankle training, Cross education, Balance, Isokinetic exercise, Ankle muscle strength

\section{INTRODUCTION}

Cross-limb transfer of performance is a well-known phenomenon, whereby bilateral performance improvements are achieved after unilateral practice, and these improvements appear to reflect usedependent plasticity within the central nervous system (CNS). ${ }^{1}$ This so-called "contralateral strength training effect" or "cross education" is task specific and occurs in opposite, homologous muscles. ${ }^{2-4}$ Cross education effects have been extensively used in studies on strength/resistance training protocols. ${ }^{5}$ Despite marginal contributions from peripheral/physiologic adaptation, strength training induces cross-effects by increasing neural drive to muscles, and altering participation of commissural interneurons on the spinal cord, which excite/inhibit contralateral motor neurons. ${ }^{2,6,7}$ Unilateral strength training effects on contralateral sides have been demonstrated in previous studies for lower extremity exercise, ${ }^{8,9}$ upper ex-

Received Jun 2, 2020 Revised Jun 12, 2020

Accepted Jun 29, 2020

Corresponding author Kyung Woo Kang

E-mail zephyr0001@hanmail.net tremity exercise, ${ }^{10,11}$ and unilateral surface electrical stimulation. ${ }^{12,13}$

When standing upright an ankle strategy is usually sufficient to correct small deviations in center of mass position and is primarily adopted during less demanding balance tasks when the sway frequency is low. ${ }^{14,15}$ Ankle strategy is commonly modeled as a singlesegment inverted pendulum that allows the body to rotate about the ankle joints as a single unit. ${ }^{1516}$ Several studies have suggested the ankle joint plays a central role in postural corrections during singlelimb standing. ${ }^{17}$ In addition, studies of quiet or perturbed standing have reported dominance of ankle muscles for balance maintenance in the antero-posterior direction. ${ }^{18}$ Furthermore, it has been suggested reduced ankle muscle strength contributes to loss of balance, ${ }^{19}$ and that enhancement of ankle muscle strength could lead to improvements in balance recovery during standing perturbations. ${ }^{20}$

Recent investigations have suggested supraspinal commands play an important role in adaptations to unilateral training, and there-

Copylight (C)2020 The Korean Society of Physical Therapy

This is an Open Access article distribute under the terms of the Creative Commons Attribution Non-commercial License (https:// creativecommons.org/license/by-nc/4.o.) which permits unrestricted non-commercial use, distribution, and reproduction in any medium, provided the original work is properly cited. 
fore, that these neural adaptations may be transferred to the untrained limb via superior levels of the CNS. ${ }^{21,22}$ In addition, crosseducation after unilateral ankle training may provide an alternative model for strength preservation in an immobilized or affected limb after stoke. However, cross-education after unilateral ankle training has been poorly addressed in the literature. Therefore, the aim of the present study was to investigate whether 4 weeks of unilateral ankle isokinetic training could enhance ankle muscle strength and balance ability of the contralateral lower extremity.

\section{METHODS}

\section{Participants}

Thirty healthy, right-handed university students volunteered for this study; the Edinburgh handedness inventory was used to assess handedness. None of the subjects had participated in any kind of strength training exercise during the previous 6 months, and all were free of any peripheral or neurological impairment that might have influenced independent single limb stance, such as, a history of fracture or surgery to a lower limb, a ligamentous ankle injury, or vestibular impairment. All subjects understood the purpose of this study and provided written, informed consent, and the experimental procedures, conformed to the Declaration of Helsinki.

\section{Procedure}

The 30 subjects were randomly and equally assigned to either a training group or a control group. Subjects in the training group $(\mathrm{n}=15)$ underwent a 4-week, right ankle, strength training program, whereas subjects in the control group $(n=15)$ did not performed any type of training. All subjects participated in a testing session before and after the 4-week intervention period.

\section{1) Strength}

Unilateral ankle strengthening training and strength assessment was performed using the Biodex 3PRO System (Biodex, Inc., Shirley, NY, USA). Training targeted ankle dorsiflexion, plantar flexion, inversion and eversion was performed on dominant sides (right sides) in five sets of 10 repetitions at an angular velocity of $60 \%$ with a rest period of 2 minutes between sets. These velocities were chosen because most daily activities are related to the ability to generate power at low velocities. ${ }^{23}$ Isokinetic dynamometer results have been previ- ously shown to produce reliable measurements of isokinetic strength. ${ }^{24}$ For ankle strengthening training, each subject was seated in the Biodex chair in an upright position with the back of the seat tilted at an angle of $85^{\circ}$. Stabilization was provided by two shoulder straps that crossed the subject's chest, a waist strap, and a thigh strap. The lateral femoral epicondyle was aligned with the axis of rotation of the dynamometer. The length of the attachment was adjusted to ensure that the ankle pad rested comfortably above lateral and medial malleoli. Range of motion was determined individually by each subject. Subject of the training group received training five times per week for four consecutive weeks, whereas in control group attended health education programs on fall prevention, balance, and exercise, and were given general information regarding health promotion for one hour per week during the 4-week study period. In addition, subjects of the control group were asked to maintain their physical activity levels for the 4-week period and to not participate in any strengthening exercise program.

\section{2) Balance}

For the one leg standing balance test, the subjects in both groups were assessed using a commercial balance device, the Biodex Stability System (Biodex, Inc., Shirley, NY, USA), a movable balance platform that provides up to $20^{\circ}$ of surface tilt in all directions. Postural stability was quantified using APSI, MLSI, and OSI stability index. Contralateral leg standing balance was assessed using the Biodex Stability System for 20 seconds and rated using a 12-point scale (level 12 is the most stable, 1 is the least stable). Subjects were initially instructed to stand on the untrained left leg with the right knee flexed with arms placed across the chest while looking straight ahead at a monitor, and to remain as motionless as possible and then to lift the right knee and flex it to $90^{\circ}$. Subjects were allowed to practice this procedure once during assessment and then two measurements were made. The mean values of two trial were entered into the analysis.

\section{Data analysis}

Statistical analyses were performed using SPSS version 18.0. The independent t-test was used to determine the significances of differences between the training and control groups in terms of baseline data (age, height, weight, and foot length). The Shapiro-Wilk test was used to check distribution normalites, and two-way repeated- 
measures analysis of variance (ANOVA) was used to assess the impact of ipsilateral ankle strength training on contralateral ankle strength and balance. Statistical significance was accepted for $\mathrm{p}$ value $<0.05$.

\section{RESULTS}

All subjects completed training and assessments and no subject

Table 1.The general characteristics of subjects

\begin{tabular}{lcc}
\hline & $\begin{array}{c}\text { Training group } \\
(n=15)\end{array}$ & $\begin{array}{c}\text { Control group } \\
(n=15)\end{array}$ \\
\hline Male/Female & $5 / 10$ & $4 / 11$ \\
Age (yr) & $23.40 \pm 2.03$ & $23.13 \pm 1.85$ \\
Height & $165.53 \pm 5.70$ & $163.33 \pm 9.63$ \\
Weight & $55.53 \pm 6.36$ & $57.13 \pm 12.13$ \\
Foot Length & $245.27 \pm 14.05$ & $242.00 \pm 18.01$ \\
\hline
\end{tabular}

reported any discomfort during the study period. No significant intergroup differences in gender distributions, ages, heights, or foot lengths were observed. Details of demographic variables are presented in Table 1.

Tables 2 and 3 summarize changes in isokinetic average torque of ipsi- and contralateral ankles for dorsiflexion, plantarflexion, inversion, and eversion according to pre- and post-intervention in the training and control groups. Two-way ANOVA with repeated measures showed significantly large main effects for group $(\mathrm{p}<0.05)$, time $(\mathrm{p}<0.05)$, and group-by-time interaction $(\mathrm{p}<0.05)$. Statistical analysis indicated that isokinetic average torque values of ipsi- and contralateral ankle in the training group were significantly higher post-intervention. Average torques value of ipsi- and contralateral ankles in the training group were $31-36 \%$ and $18-26 \%$ higher, respectively, after intervention. Stability value from pre-test to posttest for isokinetic average torque value of ipsi- and contralateral an-

Values represent mean \pm SD

Table 2. Means $( \pm S D$ ) of isokinetic average torque of ipsilateral ankles in the training and control group

\begin{tabular}{|c|c|c|c|c|c|c|c|}
\hline \multirow{3}{*}{ Parameters } & & & \multirow{2}{*}{\multicolumn{2}{|c|}{ Control group $(n=15)$}} & \multirow{3}{*}{$\begin{array}{c}\text { Group } \times \text { Time } \\
\text { F-value } \\
\text { p-value }\end{array}$} & \multicolumn{2}{|c|}{ Change values } \\
\hline & \multicolumn{2}{|c|}{ Training group $(n=15)$} & & & & Training group & Control Group \\
\hline & Pre & Post & Pre & Post & & Post-Pre & Post-Pre \\
\hline Plantarflexion & $19.63 \pm 6.17$ & $26.83 \pm 7.39^{*+}$ & $20.91 \pm 8.08$ & $21.16 \pm 7.45$ & $\begin{array}{c}F(1,28)=22.75 \\
p<0.001\end{array}$ & $7.20 \pm 4.18$ & $0.26 \pm 3.78$ \\
\hline Dorsiflexion & $8.88 \pm 3.62$ & $11.98 \pm 4.63^{*+}$ & $8.82 \pm 4.58$ & $9.28 \pm 4.40$ & $\begin{array}{c}F(1,28)=16.68 \\
p=0.005\end{array}$ & $3.10 \pm 3.19$ & $0.46 \pm 1.10$ \\
\hline Eversion & $6.09 \pm 2.42$ & $8.13 \pm 2.37^{*+}$ & $6.69 \pm 3.48$ & $6.93 \pm 3.49$ & $\begin{array}{c}F(1,28)=4.55 \\
p=0.042\end{array}$ & $2.04 \pm 3.10$ & $0.25 \pm 1.01$ \\
\hline Inversion & $6.12 \pm 1.64$ & $8.03 \pm 2.32^{*+}$ & $6.52 \pm 1.21$ & $6.82 \pm 2.21$ & $\begin{array}{c}F(1,28)=7.48 \\
p=0.011\end{array}$ & $1.91 \pm 1.40$ & $0.30 \pm 1.81$ \\
\hline
\end{tabular}

Values represent mean \pm SD.

* significant difference between pre- and post-test ( $\left.{ }^{*} p<0.05\right)$, ${ }^{+}$significant difference compared with the control group ( $\left.p<0.05\right)$.

Table 3. Means $( \pm S D)$ of isokinetic average torque of contralateral ankles in the training and control group

\begin{tabular}{|c|c|c|c|c|c|c|c|}
\hline \multirow{3}{*}{ Parameters } & & & \multirow{2}{*}{\multicolumn{2}{|c|}{ Control group $(n=15)$}} & \multicolumn{3}{|c|}{ Change values } \\
\hline & \multicolumn{2}{|c|}{ Training group $(n=15)$} & & & \multirow{2}{*}{$\begin{array}{c}\text { Group } \times \text { Time } \\
\text { F-value } \\
\text { p-value }\end{array}$} & \multirow{2}{*}{$\begin{array}{c}\begin{array}{c}\text { Training group } \\
(n=15)\end{array} \\
\text { Post-Pre }\end{array}$} & \multirow{2}{*}{$\begin{array}{c}\begin{array}{c}\text { Control Group } \\
(n=15)\end{array} \\
\text { Post-Pre }\end{array}$} \\
\hline & Pre & Post & Pre & Post & & & \\
\hline Plantarflexion & $18.33 \pm 8.63$ & $22.73 \pm 6.21^{*+}$ & $19.25 \pm 6.85$ & $20.08 \pm 7.31$ & $\begin{array}{c}F(1,28)=6.10 \\
p=0.020\end{array}$ & $4.40 \pm 4.74$ & $0.83 \pm 2.99$ \\
\hline Dorsiflexion & $8.42 \pm 3.06$ & $10.64 \pm 3.67^{\star+}$ & $9.11 \pm 3.92$ & $9.26 \pm 4.09$ & $\begin{array}{c}F(1,28)=10.95 \\
p=0.003\end{array}$ & $2.23 \pm 1.81$ & $0.15 \pm 1.62$ \\
\hline Eversion & $5.87 \pm 1.67$ & $6.97 \pm 1.85^{*+}$ & $5.99 \pm 1.76$ & $6.25 \pm 1.86$ & $\begin{array}{c}F(1,28)=4.63 \\
p=0.040\end{array}$ & $1.11 \pm 0.48$ & $0.26 \pm 1.45$ \\
\hline Inversion & $6.18 \pm 2.06$ & $7.45 \pm 2.30^{*+}$ & $6.46 \pm 2.83$ & $6.54 \pm 3.06$ & $\begin{array}{c}F(1,28)=5.25 \\
p=0.030\end{array}$ & $1.27 \pm 1.69$ & $0.08 \pm 1.07$ \\
\hline
\end{tabular}

Values represent mean \pm SD.

* significant difference between pre-and post-test $(p<0.05),{ }^{\dagger}$ significant difference compared with the control group ( $\left.p<0.05\right)$. 
Table 4. Comparison of the contralateral balance abilities of the training and control groups

\begin{tabular}{|c|c|c|c|c|c|c|c|}
\hline \multirow{3}{*}{ Parameters } & \multirow{2}{*}{\multicolumn{2}{|c|}{ Training group $(n=15)$}} & \multirow{2}{*}{\multicolumn{2}{|c|}{ Control group $(n=15)$}} & \multirow{3}{*}{$\begin{array}{c}\text { Group } \times \text { Time } \\
\text { F-value } \\
\text { p-value }\end{array}$} & \multicolumn{2}{|c|}{ Change values } \\
\hline & & & & & & \multirow{2}{*}{$\begin{array}{c}\text { Training group } \\
(n=15)\end{array}$} & \multirow{2}{*}{$\begin{array}{c}\begin{array}{c}\text { Control Group } \\
(n=15)\end{array} \\
\text { Post-Pre }\end{array}$} \\
\hline & Pre & Post & Pre & Post & & & \\
\hline APSI & $0.56 \pm 0.17$ & $0.41 \pm 0.14^{*+}$ & $0.53 \pm 0.19$ & $0.53 \pm 0.20$ & $\begin{array}{c}F(1,28)=17.55 \\
p<0.001\end{array}$ & $-0.15 \pm 0.08$ & $0.00 \pm 0.11$ \\
\hline MLSI & $0.61 \pm 0.23$ & $0.45 \pm 0.21^{*+}$ & $0.61 \pm 0.21$ & 0.59 .0 .23 & $\begin{array}{c}F(1,28)=10.92 \\
p=0.003\end{array}$ & $-0.16 \pm 0.10$ & $-0.01 \pm 0.14$ \\
\hline OSI & $0.88 \pm 0.26$ & $0.63 \pm 0.23^{*+}$ & $0.87 \pm 0.24$ & $0.85 \pm 0.31$ & $\begin{array}{c}F(1,28)=9.51 \\
p=0.005\end{array}$ & $-0.25 \pm 0.06$ & $-0.02 \pm 0.25$ \\
\hline
\end{tabular}

Values represent mean \pm SD. APSI: Anterior-Posterior Stability Index, MLSI: Medial-Lateral Stability Index, OSI: Overall Stability Index.

${ }^{*}$ significant difference between pre- and post-test $(p<0.05),{ }^{\dagger}$ significant difference compared with the control group $(p<0.05)$.

kle were statistically significant greater for the training group than for the control group.

Changes in Stability Index Scores, including APSI, MLSI, and OSI, after intervention the two groups are summarized in Table 4. Two-way ANOVA with repeated measures showed significantly large main effects for group $(\mathrm{p}<0.05)$, time $(\mathrm{p}<0.05)$, and group-bytime interaction $(\mathrm{p}<0.05)$. Statistical analysis indicated that the APSI, MLSI, and OSI in the training group were significantly lower after intervention. The improvement of stability scores, such as APSI, MLSI and OSI, from pre-test to post-test were significantly different greater for the training group when comparing the control.

\section{DISCUSSION}

The present study was designed to investigate the effects of unilateral ankle isokinetic exercises on maximal strength of both ankles strength and on one-legged standing balance of the contralateral lower extremity. Unilateral ankle isokinetic exercise was found to significantly increase ankle muscle strength in trained limbs and contralateral untrained limbs after the 4 -week training program. In addition, the training group shows significant improvements in APSI, MLSI, and OSI stability index scores of the contralateral lower extremity during one leg standing balance. These results have practical implications because they demonstrate that unilateral ankle isokinetic exercise improves muscle strength of the untrained ankle and contralateral lower extremity balance ability. In addition, they show an increase of ankle muscle strength in untrained limbs increases neural drive to contralateral untrained muscles.
In this study the training group showed significant improvements in torques of plantarflexion, dorsiflexion, eversion, and inversion of both ankles. Similarly, a previous study showed that four weeks of strength training for right wrist extensors increased extension MVC for both trained and untrained wrists. ${ }^{25}$ In addition, Shima et al. ${ }^{9}$ reported a small but significant increase in voluntary activation of both trained and untrained plantar flexors after 6 weeks of unilateral strength training, and found that this was accompanied by an increase in voluntary activation of the untrained limb as assessed by cortical stimulation. Hortobagyi et al. ${ }^{26}$ suggested an increase in glutaminergic excitatory neurotransmitters induce by high-intensity strength training reduced interhemispheric inhibition, and reduced the activity of r-aminobutyric acid inhibitory interneurons in the ipsilateral M1, resulting in greater voluntary motor drive to the untrained limb. ${ }^{27}$ In addition, the magnitude of motor irradiation is believed to be correlated to the amount of neural drive directed to the trained limb, ${ }^{6,27}$ which may be enhanced by the force generation produced during unilateral movement. ${ }^{28}$

In the present study the training group showed significant improvements in APSI, MLSI, and OSI stability index scores of the contralateral lower extremity during one leg standing balance. Single leg stance is described as a quasi-static posture, as the body is in continuous motion and never actually achieves equilibrium, even when the task is to remain as still as possible. Several studies have indicated that balance is highly related to lower extremity force production and range of motion and strength. ${ }^{29,30}$ Horlings et al. ${ }^{31} \mathrm{dem}$ onstrated that muscle weakness leads to significant postural instability during backwards perturbations. In addition, recent studies suggest that the relation between strength training exercise and bal- 
ance may be a good indicator of dynamic stability in healthy or older adults. Accordingly, improvements in balance performance following unilateral ankle isokinetic exercises have been attributed to increases in ankle muscle strength. ${ }^{32,33}$

Our findings suggest that unilateral ankle isokinetic exercise improves ankle muscle strength of contralateral lower extremity. In addition, this improvement may have been the result of better contralateral standing balance after unilateral ankle training. However, our results should be interpreted based on consideration of potential study limitations. Most obviously our results cannot be generalized because of the small sample size. Furthermore, the limited functional benefits found to be directly associated unilateral ankle strength training may not apply to other types of contralateral training, such as, cross-limb transfer of motor learning, as different types of motor skills have been shown to be transferred more or less readily to untrained limbs. Because cross-limb performance transfer has important implications in the rehabilitation environment, its efficacy as a clinical add-on technique during rehabilitative programs needs to be addressed by future studies.

\section{REFERENCES}

1. Goodwill AM, Daly RM, Kidgell DJ. The effects of anodal-tdcs on crosslimb transfer in older adults. Clin Neurophysiol. 2015;126(11):2189-97.

2. Lee M, Carroll TJ. Cross education: Possible mechanisms for the contralateral effects of unilateral resistance training. Sports Med. 2007;37(1):114.

3. Munn J, Herbert RD, Gandevia SC. Contralateral effects of unilateral resistance training: A meta-analysis. J Appl Physiol. 2004;96(5):1861-6.

4. Zhou S. Chronic neural adaptations to unilateral exercise: Mechanisms of cross education. Exerc Sport Sci Rev. 2000;28(4):177-84.

5. Oliveira AS, Brito Silva P, Farina D et al. Unilateral balance training enhances neuromuscular reactions to perturbations in the trained and contralateral limb. Gait Posture. 2013;38(4):894-9.

6. Carroll TJ, Herbert RD, Munn J et al. Contralateral effects of unilateral strength training: Evidence and possible mechanisms. J Appl Physiol. 2006;101(5):1514-22.

7. Dragert K, Zehr EP. Bilateral neuromuscular plasticity from unilateral training of the ankle dorsiflexors. Exp Brain Res. 2011;208(2):217-27.

8. Fimland MS, Helgerud J, Solstad GM et al. Neural adaptations underlying cross-education after unilateral strength training. Eur J Appl Physiol. 2009;107(6):723-30.

9. Shima N, Ishida K, Katayama K et al. Cross education of muscular strength during unilateral resistance training and detraining. Eur J Appl Physiol. 2002;86(4):287-94.

10. Farthing JP, Borowsky R, Chilibeck PD et al. Neuro-physiological adaptations associated with cross-education of strength. Brain Topogr.
2007;20(2):77-88.

11. Farthing JP, Chilibeck PD, Binsted G. Cross-education of arm muscular strength is unidirectional in right-handed individuals. Med Sci Sports Exerc. 2005;37(9):1594-600.

12. Bezerra P, Zhou S, Crowley Z et al. Effects of unilateral electromyostimulation superimposed on voluntary training on strength and cross-sectional area. Muscle Nerve. 2009;40(3):430-7.

13. Toca Herrera JL, Gallach JE, Gomis M et al. Cross-education after one session of unilateral surface electrical stimulation of the rectus femoris. J Strength Cond Res. 2008;22(2):614-8.

14. Karlsson A, Frykberg G. Correlations between force plate measures for assessment of balance. Clin Biomech. 2000;15(5):365-9.

15. Runge CF, Shupert CL, Horak FB et al. Ankle and hip postural strategies defined by joint torques. Gait Posture. 1999;10(2):161-70.

16. Gage WH, Winter DA, Frank JS et al. Kinematic and kinetic validity of the inverted pendulum model in quiet standing. Gait Posture. 2004;19(2):124-32.

17. Tropp H, Odenrick P. Postural control in single-limb stance. J Orthop Res. 1988;6(6):833-9.

18. Fujimoto M, Hsu WL, Woollacott MH et al. Ankle dorsiflexor strength relates to the ability to restore balance during a backward support surface translation. Gait Posture. 2013;38(4):812-7.

19. Hall CD, Woollacott MH, Jensen JL. Age-related changes in rate and magnitude of ankle torque development: Implications for balance control. J Gerontol A Biol Sci Med Sci. 1999;54(10):M507-13.

20. Hess JA, Woollacott M, Shivitz N. Ankle force and rate of force production increase following high intensity strength training in frail older adults. Aging Clin Exp Res. 2006;18(2):107-15.

21. Jacobs JV, Horak FB. Cortical control of postural responses. J Neural Transm. 2007;114(10):1339-48.

22. Maki BE, Mcllroy WE. Cognitive demands and cortical control of human balance-recovery reactions. J Neural Transm. 2007;114(10):127996.

23. Ferri A, Scaglioni G, Pousson M et al. Strength and power changes of the human plantar flexors and knee extensors in response to resistance training in old age. Acta Physiol Scand. 2003;177(1):69-78.

24. Hartmann A, Knols R, Murer K et al. Reproducibility of an isokinetic strength-testing protocol of the knee and ankle in older adults. Gerontology. 2009;55(3):259-68.

25. Lee M, Gandevia SC, Carroll TJ. Unilateral strength training increases voluntary activation of the opposite untrained limb. Clin Neurophysiol. 2009;120(4):802-8.

26. Hortobagyi T, Richardson SP, Lomarev M et al. Interhemispheric plasticity in humans. Med Sci Sports Exerc. 2011;43(7):1188-99.

27. Hortobagyi T. Cross education and the human central nervous system. IEEE Eng Med Biol Mag. 2005;24(1):22-8.

28. Perez MA, Cohen LG. Mechanisms underlying functional changes in the primary motor cortex ipsilateral to an active hand. J Neurosci. 2008;28(22):5631-40.

29. Lowes LP, Westcott SL, Palisano RJ et al. Muscle force and range of motion as predictors of standing balance in children with cerebral palsy. Phys Occup Ther Pediatr. 2004;24(1-2):57-77.

30. Wolfson L, Judge J, Whipple R et al. Strength is a major factor in balance, gait, and the occurrence of falls. J Gerontol A Biol Sci Med Sci. 1995; 
TRPT $\begin{aligned} & \text { The Journal of } \\ & \text { Korean Physical Therapy }\end{aligned}$

50:64-7.

31. Horlings CG, Kung UM, Van Engelen BGM et al. Balance control in patients with distal versus proximal muscle weakness. Neuroscience. 2009;164(4):1876-86.

32. Bird ML, Hill K, Ball M et al. Effects of resistance- and flexibility-exercise
Sung Min Son, et al.

interventions on balance and related measures in older adults. J Aging Phys Act. 2009;17(4):444-54.

33. Gstottner M, Neher A, Scholtz A et al. Balance ability and muscle response of the preferred and nonpreferred leg in soccer players. Motor Control. 2009;13(2):218-31. 